$\mathrm{e}^{+} \mathrm{e}^{-}$Collisions from Phi to Psi 2013 (PHIPSI2013)

International Journal of Modern Physics: Conference Series

Vol. 35 (2014) 1460430 (5 pages)

(C) The Author

DOI: $10.1142 / \mathrm{S} 201019451460430 \mathrm{X}$

\title{
HEAVY QUARK SPECTROSCOPY AT LHCb
}

\author{
SIMON EIDELMAN \\ for the $\mathrm{LHCb}$ Collaboration \\ Budker Institute of Nuclear Physics SB RAS, Novosibirsk 630090, Russia \\ and Novosibirsk State University, Novosibirsk 630090, Russia \\ Published 18 December 2014
}

\begin{abstract}
We report recent results related to spectroscopy of heavy quarks obtained with the $\mathrm{LHCb}$ detector. They include a study of the $X(3872)$ produced in continuum and $B$ decays, precise measurements of the $D$ and $D_{s}$ masses, a study of the properties of $D_{J}$ mesons and excited $B_{s}$ states as well as the first observation of the $B_{c}^{+} \rightarrow B_{s}^{0} \pi^{+}$decay.
\end{abstract}

Keywords: $p p$ collisions; $c$ and $b$ quarks.

PACS Numbers: 14.40.Rt, 14.40.Lb, 14.40.Nd

\section{Introduction}

$\mathrm{LHCb},{ }^{1}$ the detector at the LHC $p p$ collider, is a single-arm forward spectrometer at $2<\eta<5$ for a study of particles with $b$ or $c$ quarks. The integrated luminosity it collected is $37 \mathrm{pb}^{-1}$ and $1.0 \mathrm{fb}^{-1}$ at $7 \mathrm{TeV}$ (in 2010 and 2011, respectively) and $2 \mathrm{fb}^{-1}$ at $8 \mathrm{TeV}$. In this talk we briefly describe several recent analyses related to spectroscopy of heavy $c$ and $b$ quarks.

\section{Study of the $X(3872)$ State}

LHCb performed its first study of the $X(3872) \rightarrow J / \psi \pi^{+} \pi^{-}$using $34.7 \mathrm{pb}^{-1}$ of integrated luminosity collected at $\sqrt{s}=7 \mathrm{TeV} .{ }^{2}$ They measured the cross section of its production in continuum $\sigma\left(p p \rightarrow X+\right.$ anything) $\mathcal{B}\left(X \rightarrow J / \psi \pi^{+} \pi^{-}\right)=5.4 \pm$ 1.3 (stat) \pm 0.8 (syst) nb at $2.5<\eta<4.5,5<p_{T}<20 \mathrm{GeV} / c$ and observe a clear signal of the $X(3872)(565 \pm 62)$ in addition to the $\psi(2 S)(3998 \pm 83)$, see Fig. 1 . For both particles the values of the mass they obtain are consistent with those in the most precise previous measurements (KEDR for $\psi(2 S)^{3}$ and CDF for $X(3872)^{4}$ ).

In their second study they use a data sample of $1 \mathrm{fb}^{-1}$ to select $313 \pm 26$ events of $B^{+} \rightarrow X(3872) K^{+}, X(3872) \rightarrow J / \psi \pi^{+} \pi^{-}$. They perform a 5-dimensional analysis

This is an Open Access article published by World Scientific Publishing Company. It is distributed under the terms of the Creative Commons Attribution 3.0 (CC-BY) License. Further distribution of this work is permitted, provided the original work is properly cited. 


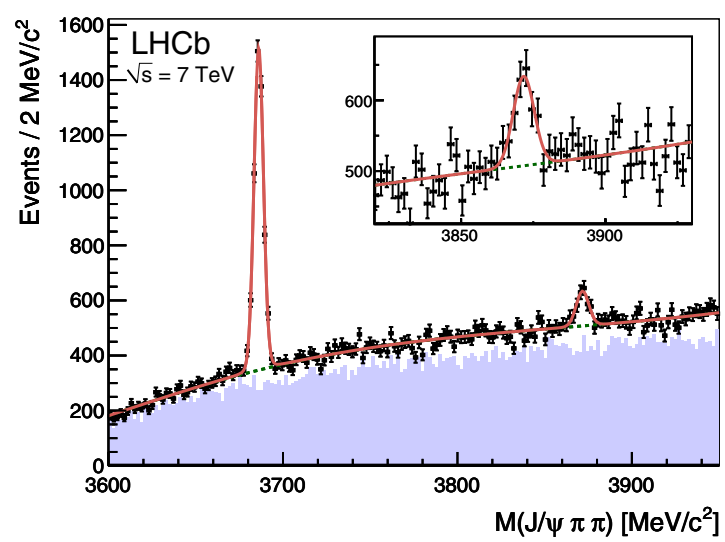

Fig. 1. Distribution of the $J / \psi \pi^{+} \pi^{-}$mass

of the angular space and come to the unambiguous conclusion about the quantum numbers of the $X(3872): J^{P}=1^{+} .^{5}$

With a data sample of $3 \mathrm{fb}^{-1}$ at 7 and $8 \mathrm{TeV}$ they study $B^{+} \rightarrow K^{+} \mu^{+} \mu^{-}$decays and select 1830 candidates in the low recoil region $\left(m_{\mu^{+} \mu^{-}}>3770 \mathrm{MeV}\right)$. In the $m_{\mu^{+} \mu^{-}}$spectrum they observe a structure with more than $6 \sigma$ significance, which properties (mass of $4191_{-8}^{+9} \mathrm{MeV}$ and width of $65_{-16}^{+22} \mathrm{MeV}$ ) are consistent with the $\psi(4160)$. This is the first observation of the $\psi(4160) \rightarrow \mu^{+} \mu^{-}$decay. $^{6}$

\section{Study of $D$ and $D_{s J}$ Mesons}

3 - and 4-body decays of charm mesons produced in semileptonic $b$-hadron decays were studied in $p p$ collisions at $\sqrt{s}=7 \mathrm{TeV}$ with $1 \mathrm{fb}^{-1}-D^{0} \rightarrow K^{+} K^{-} \pi^{+} \pi^{-}$, $D^{0} \rightarrow K^{+} K^{-} K^{-} \pi^{+}, D^{+} \rightarrow K^{+} K^{-} \pi^{+}$and $D_{s}^{+} \rightarrow K^{+} K^{-} \pi^{+}$. These modes have a relatively low energy release, the difference between the mass of the $D$ meson and the sum of the masses of the daughter particles, resulting in reduced systematic uncertainties due to the calibration of the momentum scale and thus a high-precision measurement of the $D^{0}, D^{ \pm}$and $D_{s}^{+}$mass and mass differences. ${ }^{7}$ As an example, Fig. 2 shows the invariant mass distributions for the $K^{+} K^{-} \pi^{+} \pi^{-}$and $K^{+} K^{-} K^{-} \pi^{+}$ final states together with the corresponding fits.

The results of the fits are used to determine $M\left(D^{0}\right), M\left(D^{+}\right)-M\left(D^{0}\right)$ and $M\left(D_{s}^{+}\right)-M\left(D^{+}\right)$shown in Table 1 , which also lists the best previous measurements as well as the results of the PDG fit. ${ }^{11}$ Including the result for the $D^{0}$ mass in the determination of the $X(3872)$ binding energy gives $E_{B}=M\left(D^{0} D^{* 0}\right)-$ $M(X(3872))=0.09 \pm 0.28 \mathrm{MeV}$ and reinforces the conclusion that if the $X(3872)$ is a molecule, it is extremely loosely bound.

These measurements, together with others listed by PDG, can be used to determine a more precise value of the $D_{s}^{+}$mass, $M\left(D_{s}^{+}\right)=1968.19 \pm 0.20 \pm 0.14 \pm 0.08$ 
Table 1. Measurement of the $D$ and $D_{s}$ masses

\begin{tabular}{crrr}
\hline Quantity & \multicolumn{1}{c}{$\begin{array}{c}\text { LHCb } \\
\text { measurement }\end{array}$} & $\begin{array}{c}\text { Best previous } \\
\text { measurement }\end{array}$ & \multicolumn{1}{c}{ PDG fit } \\
\hline$M\left(D^{0}\right)$ & $1864.75 \pm 0.19$ & $1864.85 \pm 0.18$ CLEO $^{8}$ & $1864.86 \pm 0.13$ \\
$M\left(D^{+}\right)-M\left(D^{0}\right)$ & $4.76 \pm 0.14$ & $4.7 \pm 0.3 \quad$ MARK2 $^{9}$ & $4.76 \pm 0.10$ \\
$M\left(D_{s}^{+}\right)-M\left(D^{+}\right)$ & $98.68 \pm 0.05$ & $98.4 \pm 0.3 \quad$ BaBar $^{10}$ & $98.88 \pm 0.25$ \\
\hline
\end{tabular}

$\mathrm{MeV}$, which is more precise than that obtained from the fit to open charm mass data $M\left(D_{s}^{+}\right)=1968.49 \pm 0.32 \mathrm{MeV} .^{11}$

A study of $D^{+} \pi^{-}, D^{0} \pi^{+}$and $D^{*+} \pi^{-}$final states is performed using a data sample of $1.0 \mathrm{fb}^{-1}$ collected at $7 \mathrm{TeV}$. The $D_{1}(2420)^{0}$ resonance is observed in the $D^{*+} \pi^{-}$final state and the $D_{2}^{*}(2460)$ resonance is observed in the $D^{+} \pi^{-}, D^{0} \pi^{+}$and $D^{*+} \pi^{-}$final states. For both resonances, their properties and spin-parity assignments are obtained. In addition, two natural parity and two unnatural parity resonances are observed in the mass region between 2500 and $2800 \mathrm{MeV}$. Further structures in the region around $3000 \mathrm{MeV}$ are observed in all the $D^{+} \pi^{-}, D^{0} \pi^{+}$and $D^{*+} \pi^{-}$final states.

\section{Decays of $B_{s}^{0}$ and $B_{c}$}

Orbitally excited $(L=1) B_{s}^{0}$ states were studied at $7 \mathrm{TeV}$ with $1.0 \mathrm{fb}^{-1} .{ }^{14}$ Figure 3 shows the mass difference distribution $m\left(B^{+} K^{-}\right)-m\left(B^{+}\right)-m\left(K^{-}\right)$. The left and right peaks correspond to the previously observed decays $B_{s 1} \rightarrow B^{*+} K^{-}$and
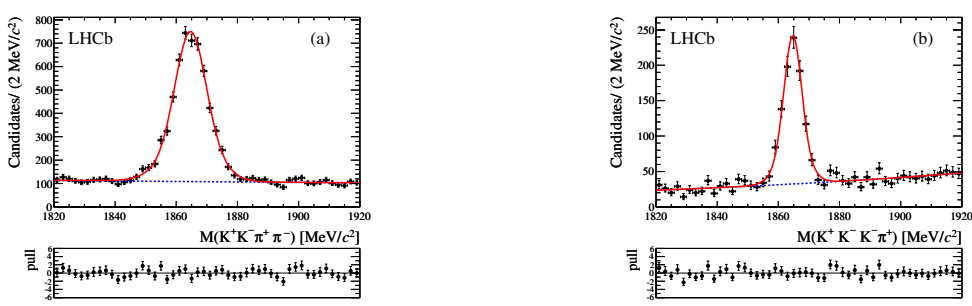

Fig. 2. Invariant mass distributions for the (a) $K^{+} K^{-} \pi^{+} \pi^{-}$and (b) $K^{+} K^{-} K^{-} \pi^{+}$final states.

Table 2. Polarization properties of the $D_{J}$ resonances

\begin{tabular}{ccc}
\hline Resonance & $J^{P}$ (Function) & $h$ parameter \\
\hline$D_{1}(2420)^{0}$ & $1^{+}\left(1+h \cos ^{2} \theta_{\mathrm{H}}\right)$ & $3.30 \pm 0.48$ \\
$D_{2}^{*}(2460)^{0}$ & $2^{+}\left(\sin ^{2} \theta_{\mathrm{H}}\right)$ & - \\
$D_{J}^{*}(2650)^{0}$ & Nat. $\left(\sin ^{2} \theta_{\mathrm{H}}\right)$ & - \\
$D_{J}^{*}(2760)^{0}$ & Nat. $\left(\sin ^{2} \theta_{\mathrm{H}}\right)$ & - \\
$D_{J}(2580)^{0}$ & Unnat. $\left(1+h \cos ^{2} \theta_{\mathrm{H}}\right)$ & $4.2 \pm 1.3$ \\
$D_{J}(2740)^{0}$ & Unnat. $\left(1+h \cos ^{2} \theta_{\mathrm{H}}\right)$ & $3.1 \pm 2.2$ \\
$D_{J}(3000)^{0}$ & Unnat. $\left(1+h \cos ^{2} \theta_{\mathrm{H}}\right)$ & $1.5 \pm 0.9$ \\
\hline
\end{tabular}




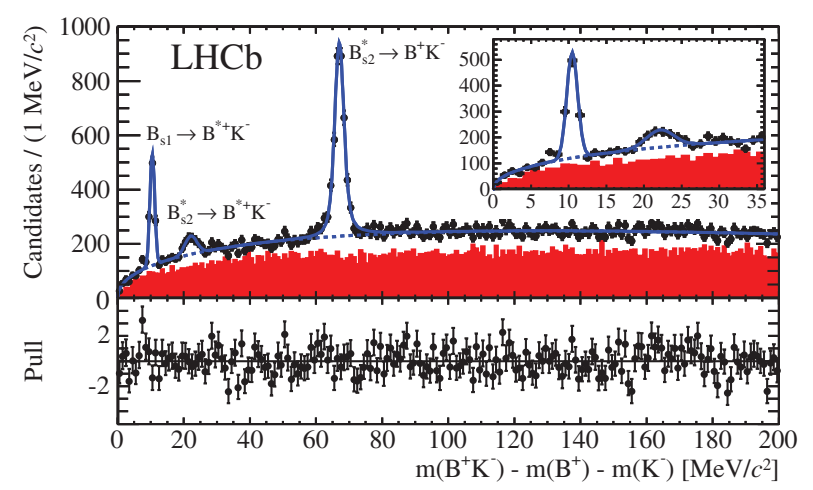

Fig. 3. Mass difference distribution $m\left(B^{+} K^{-}\right)-m\left(B^{+}\right)-m\left(K^{-}\right)$

$B_{s 2}^{*} \rightarrow B^{+} K^{-}$, respectively, whereas that in the middle is identified as a new decay mode $B_{s 2}^{*} \rightarrow B^{*+} K^{-}$.

A fit of the distribution gives the values of the difference from which, using $m\left(B^{+}\right), m\left(B_{s 1}\right)$ and $m\left(B^{*+}\right)-m\left(B^{+}\right)$, one obtains:

$$
\begin{gathered}
m\left(B^{*}\right)=5324.26 \pm 0.30 \pm 0.23 \pm 0.17 \mathrm{MeV} \\
m\left(B_{s 1}\right)=5828.40 \pm 0.04 \pm 0.04 \pm 0.41 \mathrm{MeV} \\
m\left(B_{s 2}^{*}\right)=5839.99 \pm 0.05 \pm 0.11 \pm 0.17 \mathrm{MeV}
\end{gathered}
$$

The more precise value of the $m\left(B^{*+}\right)$ mass allows to determine that $m\left(Z_{b}(10610) a t^{+}\right)$and $m\left(Z_{b}(10650)^{+}\right)$are $3.69 \pm 2.05 \mathrm{MeV}$ and $3.68 \pm 1.71 \mathrm{MeV}$ above the $B \bar{B}^{*}$ and $B^{*} \bar{B}^{*}$ thresholds. One more important result is the first measurement of the $B_{s 2}^{*}$ width. Finally, the observation of $B_{s 2}^{*} \rightarrow B^{*+} K^{-}$and $B_{s 2}^{*} \rightarrow B^{+} K^{-}$favors $J^{P}=2^{+}$.

The $B_{c}^{+}$meson is the ground state of the $\bar{b} c$ system, the only weakly decaying doubly heavy meson. All $B_{c}^{+}$meson decays known to date are decays in which the constituent $b$ quark decays weakly to a $c$ quark. It is interesting to look for a decay of the $B_{c}^{+}$meson to another $B$ meson with the bottom quark acting as a spectator. For this search $\mathrm{LHCb}$ used a data sample of $p p$ collisions corresponding to an integrated luminosity of $1 \mathrm{fb}^{-1}$ taken at a center-of-mass energy of $7 \mathrm{TeV}$, and $2 \mathrm{fb}^{-1}$ taken at $8 \mathrm{TeV} .{ }^{15}$ As a result of the analysis, the decay $B_{c}^{+} \rightarrow B_{s}^{0} \pi^{+}$has been observed using two decay modes of the $B_{s}^{0}: B_{s}^{0} \rightarrow D_{s}^{-} \pi^{+}, D_{s}^{-} \rightarrow K^{+} K^{-} \pi^{-}$with a $7.5 \sigma$ significance and $B_{s}^{0} \rightarrow J / \psi \phi, J / \psi \rightarrow \mu^{+} \mu^{-}, \phi \rightarrow K^{+} K^{-}$with a $5.5 \sigma$ significance, see Fig. 4. This is the first observation of the weak decay of a $B$ meson decaying to another $B$, with the $b$ quark acting as a spectator

\section{Summary}

- $X(3872)$ is clearly selected both in continuum and $B$ decays

- $X(3872)$ quantum numbers are $1^{+}$ 

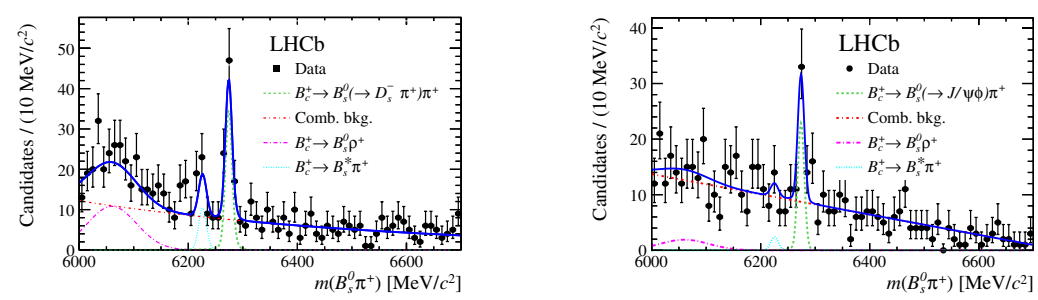

Fig. 4. $\quad B_{s}^{0} \pi^{+}$invariant mass distribution for the (a) $B_{s}^{0} \rightarrow D_{s}^{-} \pi^{+}$and (b) $B_{s}^{0} \rightarrow J / \psi \phi$

- Precise determination of $D$ meson masses

- Various $D_{J}$ were observed with $J^{P}$ analysis

- Excited $B_{s}$ states were studied

- Decay $B_{c}^{+} \rightarrow B_{s}^{0} \pi^{+}$was observed

- Due to the high cross section, thus huge statistics, and excellent performance $\mathrm{LHCb}$ has a high potential for spectroscopy studies

\section{Acknowledgments}

The author is grateful to the Workshop organizers for the interesting program and kind hospitality, to Novosibirsk State University for support and to his LHCb colleagues for useful discussions. This work is supported by the Ministry of Education and Science of the Russian Federation, the RFBR grant 12-02-01296 and the DFG grant HA 1457/9-1.

\section{References}

1. A.Augusto Alves, Jr. et al., JINST 3, S08005 (2008).

2. LHCb Collab. (R. Aaij et al.), Eur. Phys. J. C 72, 1972 (2012).

3. KEDR Collab. (V.V. Anashin et al.), Phys. Lett. 711, 280 (2012).

4. CDF Collab. (T. Aaltonen et al.), Phys. Rev. Lett. 103, 152001 (2009).

5. LHCb Collab. (R. Aaij et al.), Phys. Rev. Lett. 110, 222001 (2013).

6. LHCb Collab. (R. Aaij et al.), Phys. Rev. Lett. 111, 112003 (2013).

7. LHCb Collab. (R. Aaij et al.), JHEP 1306, 065 (2013).

8. CLEO Collab. (C. Cawlfield et al.), Phys. Rev. Lett. 98, 092002 (2007).

9. MARK2 Collab. (R.H. Schindler et al.), Phys. Rev. D 24, 78 (1981).

10. BaBar Collab. (B. Aubert et al.), Phys. Rev. D 78, 011103 (2008).

11. Particle Data Group (J. Beringer et al.), Phys. Rev. D 86, 010001 (2012).

12. LHCb Collab. (R. Aaij et al.), JHEP 1309, 145 (2013).

13. BaBar Collab. (P. del Amo Sanchez et al.), Phys. Rev. D 82, 111101 (2010).

14. LHCb Collab. (R. Aaij et al.), Phys. Rev. Lett. 110, 151803 (2013).

15. LHCb Collab. (R. Aaij et al.), Phys. Rev. Lett. 111, 181801 (2013). 